\title{
THE FEASIBILITY OF THE X-RAY LASER PUMPED WITH A NUCLEAR EXPLOSION
}

David W. Hafemeister

Program in Science and Technology for International Security Physics Department, M.I.T., Cambridge, MA 02139

\section{Abstract}

Recent reports have indicated that an x-ray laser pumped with a nuclear explosion at a wavelength of $1.4 \mathrm{~nm}(0.9 \mathrm{keV})$ has made some initial progress. It has been proposed to extend the development of this device to establish it as part of a layered ballistic missile defensive system to protect the United States against a nuclear attack. This paper will discuss the following aspects on the feasibility of this system: (l) Conditions affecting the efficiency of the X-ray laser; (2) Angular resolution and size of the laser rods; (3) Energy on targets; (4) Basing Modes; (5) Countermeasures; and (6) Legal constraints.

\section{Introduction}

The subject of $x$-ray lasers has been discussed by several authors ${ }^{1-5}$, but the feasibility of pumping an x-ray laser with a nuclear explosion has not been widely discussed. The Defense Technologies Study ${ }^{6,7}$, headed by James Fletcher, and other references have indicated that the $x$-ray laser pumped with a nuclear explosion has been tested with some initial progress and that it is presently being considered as a candidate for the U.S. ballistic missile defense system ${ }^{9}$ (BMD).

About $70 \%$ of the energy of a nuclear device exploded in space appears in the form of black-body radiation. Temperatures of the order of tens of millions of degrees $k$ acting over 1 to 10 nanoseconds produce a tremendous flux of soft $x$-rays. After interacting with the surrounding media, the $x$-rays can heat the laser rods to a high temperature plasma state by photoionizing the atoms in the rod. The population of the atomic states can be inverted by "tuning" the choice of the atoms in the rod to the temperature of the rods. A portion of the weapon's black-body radiation spectra can be amplified, but the question is to what extent this can be done. Because there are no suitable, highly reflecting, mirrors for 1 keV $x$-rays, the $x$-ray laser is a single pass laser. The laser pulse is amplified by superradiantio emission; the emission rate of an assembly of atoms can be much greater than an isolated atom. This paper will discuss several mechanisms which will tend to degrade both the efficiency and the angular resolution of the $x$-ray laser system, as well as other scientific, technical, and legal aspects which would affect its ultimate feasibility of deployment.

\section{Factors Affecting the Efficiency of the X-ray Laser}

In order to consider the possible efficiency of the $x$-ray laser, we must model the energy input "signal from the nuclear explosion. There are three key parameters that depend on the yield of a nuclear explosion ( $Y$ ): these are the temperature ( $T$ ), the duration of the explosion (dt) and the initial area of the spherical explosion (A). of course, the actual temperature rise is somewhat exponential, but for simplicity, we will assume a step function pulse of temperature $T$ over time dt. For the case of a lo kiloton warhead, about $70 \%$ of the $y$ ield is released in the form of black-body radiation:

$$
Q=(0.70)\left(4.2 \times 10^{13} \mathrm{~J}\right)=3.2 \times 10^{13} \mathrm{~J} \text {. }
$$

This energy would be radiated approximately according to the relation

$$
Q=\left(\sigma T^{4}\right)(A)(d t)
$$

under the assumption of a square pulse. Using the values of $A=100 \mathrm{~cm}^{2}$ ( $\mathrm{radius}=2.8 \mathrm{~cm}$ ), $d t=10^{-9} \mathrm{~s}$, we obtain $T=0.8 \times 10^{8} \mathrm{~K}$. If the explosion lasted $10^{-8} \mathrm{~s}$, the temperature would be $T=0.4 \times 10^{8} \mathrm{~K}$.

The $x$-rays for the laser could be obtained from transitions from the $n=4$ and 5 states to the $n=3$ states in highly ionized atoms near $z=30$. A possible candidate is zinc (see Fig. l for energy level diagrams of neutral zinc and zinc with one electron). A wide variety of charge states and electronic configurations between these two extreme cases would be present in the laser rod. The temporary population inversion between the states could result from a variety of reasons: 
Fig. 1. Energy Level Diagrams for $Z n I$ and $Z n X X X$. Many charge states of $Z n$ would exist in the laser rod; this figure displays the two extreme cases of neutral zinc ( $Z n I)$, and zinc with one electron $(Z n X X X)$. The possible $0.9 \mathrm{keV}$ transition for the $x$-ray laser is indicated on the level diagrams.

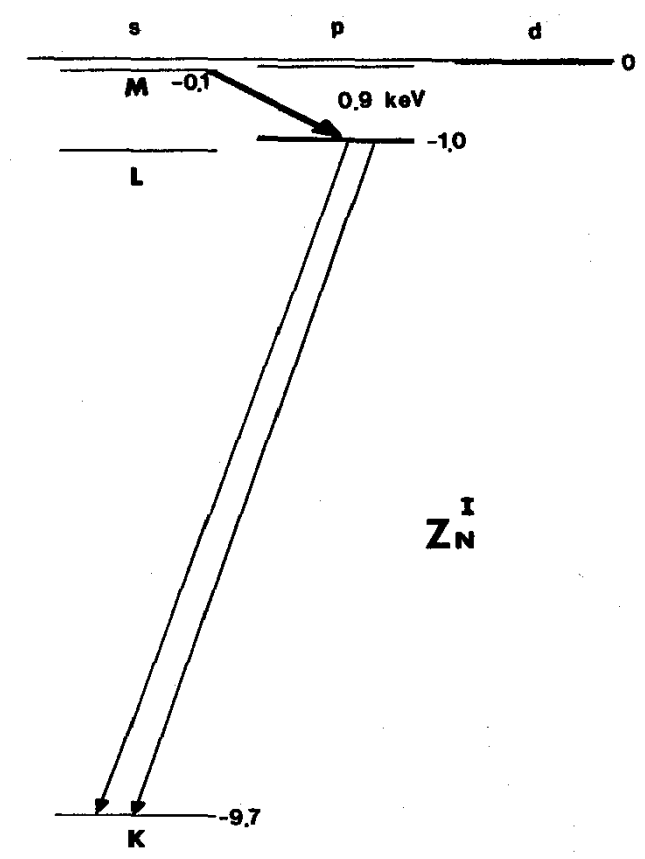

(1) Radiation Lifetimes. The excited s states would not be able to easily transition to the Is state because of selection rules; these states would be depopulated primarily by an x-ray transition to the $p$ states. The more energetic transitions would proceed more rapidly because of their greater energies. The population of the ions in the laser rod would be inverted (more holes in the p states than in the upper s states) because of these transition rates and selection rules.

(2) Photo-Ionization Probabilities. Since the peak of the black-body distribution at $0.8 \times 10^{8} \mathrm{~K}(\lambda=0.04 \mathrm{~nm}, E=30 \mathrm{keV})$, and the average energy ( $\left.3 \mathrm{kT} / 2=10 \mathrm{keV}\right)$ are considerably larger than the $x$-ray transition of about l kev, there are about loo times more photons in the rod at the higher energy transition of $10 \mathrm{keV}$ than at 1 keV. See Fig. 2 for a comparison of the black-body distribution of the number of photons ( $N$ ) as compared to the critical absorption levels ( $K, L, M)$ for neutral zinc. Since the photoionization crosssection drops rapidly with energy $\left(E^{-3}\right)$ above its absorption edges, the bath of photons will photoionize the more tightly bound states in preference to the less tightly bound (higher energy) states.

Fig. 2. The Planck distribution of the number of photons (N) vs. the photon energy (E) at $0.8 \times 10^{8} \mathrm{~K}$. The usual Planck energy distribution is

$U=(N)(E)$. The $K, L$, and M critical absorption edges of $Z n$ are shown for comparison, along with the photoionization cross-section $(\sigma)$.

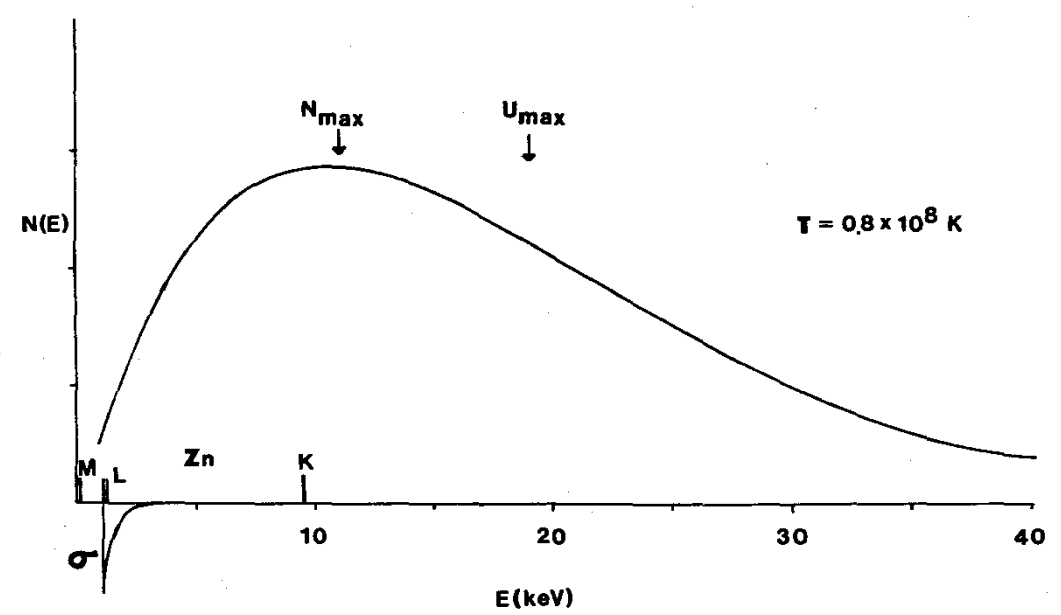


(3) Auger Transitions. Only about $50 \%$ of the KL transitions in neutral Zn take place by the emission of $x-$ rays; in about $50 \%$ of the cases an "Auger" electron" from another shell is ejected from the atom. For the case of the x-ray laser, the highly ionized atoms would be lacking many electrons, thus reducing the Auger effect. This would tend to increase the $x$-ray branching ratio and increase the residence time in the upper s state.

(4) Recycle Times. Since the lifetime of the l keV transition would be about lo-13 s, it will be necessary to repopulate this state many times over the duration of a laser pulse $\left(d t=10^{-9} \mathrm{~s}\right)$. By integrating Planck's black-body spectrum over the photoionization crosssection, we have estimated that the time to empty the p state is about $10^{-18}$ s. Thus, it is possible to empty the p state very quickly with black-body radiation after it has been filled by an s to p transition.

(5) High Temperatures Available. The "reflected heat" of the nuclear explosion can raise the temperature of the rod to very high temperatures because it is very thin; the stored energy is proportional to the radius squared, while the surface area of the rod that receives the radiation is proportional to the radius.

In contrast to the favorable conditions listed above, there are many possible causes for a diminished gain (efficiency) of the $x$-ray laser. It would be very difficult to calculate the efficiency of the $x$-ray laser from first principles. This paper will only indicate some of the serious design problems of the $x$-ray laser which would cause reduced output and angular resolution. Our calculations in Sec. V will ignore these difficulties by assuming (1) an optimistic total efficiency of $0.1 \%$ and (2) no degradation in the theoretical angular resolution of the $x$-ray beam. Some of the possible causes for a reduced output are as follows:

(1) Timing and Alignment. The velocity of the speed of sound ( $v_{s}$ ) in a $Z n$ plasma rod is very high. Under the regime of lower temperatures, the velocity of the speed of sound is

$$
v_{S}=(\gamma P / \rho)^{0.5} \cong 10^{5} \mathrm{~m} / \mathrm{s} \text {. }
$$

at $0.8 \times 10^{8} \mathrm{k}$, normal densities of zinc $(\rho=7.1 \mathrm{~g} / \mathrm{cm})$, and $\gamma=5 / 3$. Under the regime of very high temperatures, the pressure $(P)$ due to black-body radiation should predominate $\left(10^{8}\right.$ atmospheres) giving

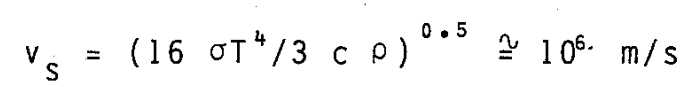

at a temperature of $0.8 \times 10^{8} \mathrm{~K}$ and normal densities. If the atomic density is increased by a factor of 100 , v will drop to about $10^{5} \mathrm{~m} / \mathrm{s}$. At any rate, the velocity of sound in this very high temperature plasma state will be quite high, of the order to $10^{5} \mathrm{~m} / \mathrm{s}$. If the laser is to maintain its alignment, the time dispersion between the heating of a particular region and the arrival of the laser pulse at that region cannot be too large. For example, if the displacement and diffusion of the rod ( $\Delta d$ ) were about lo microns, or about $15 \%$ of the rod diameter (Sec. III), one might expect to experience problems with both colinarity and uniformity of the laser rod which would complicate both aiming and beam width. It would take a discrepancy in time of about

$$
\Delta d / v_{s} \cong 10^{-5} \mathrm{~m} / 10^{5} \mathrm{~m} / \mathrm{s}=10^{-10} \text { seconds }
$$

to start to cause some problems. Since the soft $x$-ray heat pulse from the weapon re-emits from the walls in a "diffuse" rather than a specular manner, it should be quite difficult to reduce the dispersion in the timing of the rod.

(2) Stark Broadening. The linewidth of the laser pulse effects the output in two ways. A broad linewidth allows a greater portion of the input energy to be amplified, however, a broader linewidth will reduce the gain/cm of the laser.

(3) Atomic Absorption. The value of the mass absorption coefficient in neutral zinc is $2000^{\frac{\mathrm{cm}^{2}}{\mathrm{~g}} \mathrm{~g} \text { for } x-\mathrm{rays}}$ of $0.9 \mathrm{keV}$. If $0.001 \mathrm{~cm}$ (about $10^{-5}$ of the $200 \mathrm{~cm} \mathrm{rod}$ ) is in the neutral zinc state, the beam will be reduced by about one-millionth of its intensity. One would expect that the highly ionized zinc atoms would not absorb x-rays very strongly, but in practice one will have to balance these absorbing effects against the gain of the laser.

(4) Charge States and Electronic Configurations. Many ionization states of zinc are possible at $10^{8} \mathrm{~K}$; in addition, the electrons can be coupled together in a variety of ways in which the location of the atomic levels are changed significantly. One would expect large shifts in the $x$-ray energies caused by vacancies in the inner shells. Relevant data 512 has been taken on the $x$-rays that have been produced by an intensive pulse of laser light $\left(1014 \mathrm{~W} / \mathrm{cm}^{2}\right)$ on an iron slab. By using a bent crystal spectrometer, it has been 
possible to resolve the complex spectra which indicates charge configurations between neutral iron and iron ionized more than 20 times. These multiple charge and configurational states for the atoms can reduce the ultimate efficiency of an x-ray laser.

\section{Angular Resolution and Size of the $x$-ray Laser}

There are two competing optical phenomena which tend to spread the $x$-ray laser beam: (1) geometrical ray optics, and (2) diffraction broadening. These two effects act in the opposite direction. A laser rod that is too broad in diameter will tend to have a wide angular resolution dictated by simple ray optics; $\theta=D / L$, where $D$ is the diameter of the laser and $L$ is its length. A laser that is too narrow in diameter will suffer from diffraction broadening; the angular resolution from a circular aperture is $\theta=1.22 \lambda / 0$ where $\lambda$ is the wavelength of the $x$-rays. By properly combining these effects 113 , one can obtain the minimum angular resolution

$$
\theta_{m}=1.6(\lambda / L) 0.0
$$

and the diameter of the rod to obtain the minimum resolution,

$$
D_{m}=1.1(\lambda L)^{0.5}
$$

Using $\lambda=1.4 \mathrm{~nm}$ and $L=2 \mathrm{~m}, \theta_{\mathrm{m}}=4.1 \times 10^{-5}$ radians for the half angle, and $8.3 \times 10^{-5}$ for the fuli angle. The diameter of the rod needed to obtain this value of $\theta$ is $D_{m}=58$ microns; this value is similar to the diameter of $30 \mathrm{microns}$ suggested by chap.line and wood ${ }^{2}$. These authors indicate that it is quite difficult to design an $x-r a y$ laser which would allow values of $D$ much less than $(\lambda L)^{0.5}$.

These narrow rods have a very small volume of about $0.005 \mathrm{~cm}^{3}$ which would contain only about $4 \times 10^{20}$ atoms of zinc at normal densities (more when compressed). If each atom. contributed only one $x-r a y$ of $l$ kev to the pulse, this would imply a total energy of about $Q=6 \times 10^{4} \mathrm{~J}$ per pulse. Since a burst from the $x-r a y$ laser must have about $10^{11} \mathrm{~J}$ (Sec. IV), it is clear that each atom must contribute many $x$-rays to the pulse of $x$-rays. one way to enhance the process would be to use many "fibers" of Zn to develop multiple parallel paths.

\section{X-ray Energy on Target}

It is generally accepted that energy densities of about $1 \mathrm{~kJ} / \mathrm{cm}^{2}$ should be able to disable a missile booster. The Fletcher report on Defensive Technologies has used the range of 0.4 to $2 \mathrm{~kJ} / \mathrm{cm}^{2}$ for present booster hardness; other Department of Defense Studies indicate that the USSR might be able to harden their ballistic missile booster to about $20 \mathrm{~kJ} / \mathrm{cm}^{2}$. A re-entry vehicle (RV) would have a greater hardness of about $150 \mathrm{~kJ} / \mathrm{cm}^{2}$. As an initial requirement for destroying missiles in the boost phase, we will use the value of $1 \mathrm{~kJ} / \mathrm{cm}^{2}$, and then allow for an additional hardening of a factor of 20 for the booster and 150 for the RV.

The $x$-ray laser is intended to have a kill radius of about 1000 to $2000 \mathrm{~km}$. Using a fullangle width of the laser beam at $1000 \mathrm{~km}$ is $W=\left(10^{6} \mathrm{~m}\right)\left(8.3 \times 10^{-5}\right)=83 \mathrm{~m}$, which gives an area of $W=7 \times 10^{7} \mathrm{~cm}^{2}$. In order to destroy a missile booster at this distance, it would require a beam of energy of

$$
Q=\left(1000 \mathrm{~J} / \mathrm{cm}^{2}\right)\left(7 \times 10^{7} \mathrm{~cm}^{2}\right)=7 \times 10^{10} \mathrm{~J}
$$

for the unhardened booster, and $1.4 \times 10^{12} \mathrm{~J}$ for the hardened booster, and $10^{13}$ for the RV. Alternatively, this would require a beam intensity per steradian of

$$
(Q) /\left(2 \theta_{\mathrm{m}}\right)^{2}=\left(7 \times 10^{10} \mathrm{~J}\right) /\left(8.3 \times 10^{-5}\right)^{2}=10^{19} \mathrm{~J} / \mathrm{steradian}
$$

for the unhardened missile, $10^{20} \mathrm{~J} / \mathrm{steradian}$ for the hardened missile, and $10^{21} \mathrm{~J} / \mathrm{steradian}$ for the RV. These results are consistent with the report on Fletcher Report which "requires validation at moderate brightness - $10^{19} \mathrm{~J} / \mathrm{steradian,} \mathrm{plus} \mathrm{upgrading} \mathrm{to} \mathrm{three} \mathrm{orders} \mathrm{of}$ magnitude."

\section{Efficiency and Yield for the X-ray Laser}

Let us assume that there is one $x$-ray system attached to a nuclear warhead. Let us optimistically speculate that $1 \%$ of the black-body radiation is able to be contained in the laser system, and that the laser can convert $10 \%$ of that energy into the laser pulse; this gives a speculated al l-over efficiency of $0.1 \%$ of the system. If $70 \%$ of the energy of the warhead is emitted as black-body radiation, the yield of the weapon would be

$$
Y=(1.4)\left(10^{3}\right)\left(7 \times 10^{10} \mathrm{~J}\right)=10^{14} \mathrm{~J}=25 \text { kilotons, }
$$


against an unhardened booster, a yield of 500 kilotons against a hardened booster, and 4 megatons against an RV. Some drawings have indicated that the $x$-ray laser system might have as many as 50 laser weapons for 50 separate targets; then the required yields would have to be a factor of 50 times larger.

If one examines the present trend ${ }^{14}$ for the efficiency of more normal lasers, we see that the efficiency of the lasers drops with increasing photon energy. By extrapolating these curves to $1 \mathrm{keV}$, we see that one "might expect" an efficiency of between $5 \times 10^{-5}$ to $10^{-9}$. These trends do not prove the ultimate value of the efficiency of the $x$-ray laser, but it should be pointed out that these values are about 4 orders of magnitude lower than our speculated value of efficiency of $10^{-3}$. If an $x-r a y$ laser were operated in the $10 \mathrm{keV}$ region, the energy requirements would be less, but the efficiency would also, most likely, be considerably less.

\section{Basing Modes for the $X$-ray Laser}

The trajectory of an ICBM can be divided ${ }^{9}$ into three parts: (1) The boost phase which takes 2 to 5 minutes to reach altitudes of about $300 \mathrm{~km}$; (2) The midcourse phase which takes about 30 minutes, reaching a maximum altitude of about $1000 \mathrm{~km}$; and (3) the re-entry phase which takes about 0.5 to 2 minutes to re-enter the atmosphere. It is most advantageous to attack the boost phase because the missile boosters are, at present, relatively soft, the plumes from the booster give a good infrared signature, and the system has not yet launched its re-entry vehicles. Each of these phases would present a different set of problems for the deployment of the $x$-ray laser.

It would not be economically feasible to base the $x$-ray launchers permanently in space because it would be in the proper location at most $5-10 \%$ of the time. To destroy the missile during the boost phase would require forward basing in the Arctic that could respond very quickly (in a matter of minutes) to the early warning signals of the soviet missile launchings obtained by U.S. Satellites. The x-ray lasers would have to arrive on station in about a minute above the atmosphere in order to catch the ICBMs during the remainder of its boost phase. The $x$-ray laser would want to avoid absorption of the $x$-rays by the atmosphere. It would be necessary to locate other laser launchers near the coastal regions of the U.S. to combat submarine launched ballistic missiles (SLBM). Target acquisition, tracking, and pointing would be carried out with infrared technology; the precision pointing would require laser "radar" tracking to about 10 microradians (Sec. III). Alternatively, one might consider basing synthetic aperture radars on airplanes near the Soviet Union or on satellites on geosynchronous orbits. Midcourse acquisition and tracking might be carried out on space-based platforms in low Earth orbit. The $x$-ray laser would not be effective for the re-entry phase since the $x$-rays would be absorbed by the atmosphere (below about $130 \mathrm{~km}$ for $1 \mathrm{keV} x$-rays).

VII. Countermeasures

The $x$-ray laser and its basing, sensing, and guidance systems would be a technological tour de force if they could be constructed. Some countermeasures that could be applied by the opposition to circumvent the x-ray laser are as follows:

Boost Phase

(1) Harden the boosters to $20,000 \mathrm{~J} / \mathrm{cm}^{2}$ or more.

(2) Use ICBM launchers with very high acceleration in order to limit the boost phase to altitudes of less than about $130 \mathrm{~km}$. In Fig. 3 we have calculated the reduction of the $x$-ray flux as a function of the altitude of the target in the atmosphere.

Fig. 3. The relative intensity of $x$-rays ( I $\left./ \bar{I}_{0}\right)$ vs. the height of the atmisphere. The $x$-rays of $1-10 \mathrm{keV}$ are emitted above the atmosphere and penetrate into the atmosphere. We have assumed an incidence angle of $\phi=60^{\circ}$ with respect to the zenith angle. For the case of normal incidence of $\phi=0^{\circ}$, the curves would be shifted about $5 \mathrm{~km}$ to the left.

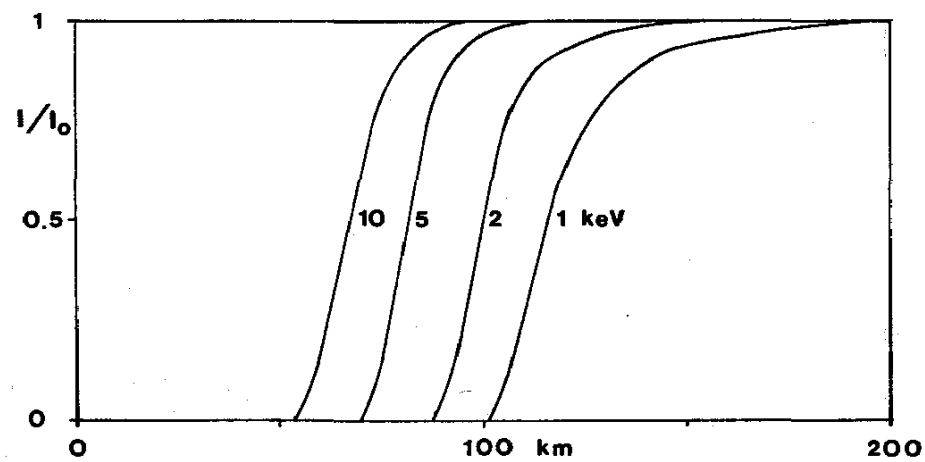


(1) Harden the bus and re-entry vehicles. Metal on mylar films could be used to stop the 1 keV x-rays. The geometry of the "skirts" can be designed to minimize the impulse delivered to the RVS.

(2) Penetration Aids could be used to foil the x-ray laser system. Some examples of penetration aids are decoys, chaff, aluminized balloons, and gaseous and aerosol absorbers.

(3) Masking the infrared signature of the Rvs by using insulators covered with materials with a low emissivity in the infrared such as black chrome. One could contemplate precooling the RVs, but that probably wouldn't be necessary. Conversely, one could slightly heat the decoys and balloons to enhance their infrared signature.

(4) Since it is unlikely that a "direct hit" could be validated by the defensive side, it would not be possible to retarget further laser pulses on the basis of target acquisition data. Imaging technologies would not be able to resolve the damage by the $x$-rays to a re-entry vehicle.

other countermeasures

(1) Attack the $x$-ray laser sensors and bases with an electromagnetic pulse (EMP) from a nuclear explosion or with other means. Use SLBMs in order to reduce the warning time.

(2) Design attack scenarios that avoided the locations of the $x$-ray laser, or that flooded a region with many RVs. It is not correct to multiply the probabilities of a layered defense since these probabilities can be dependent on each other.

VIII. Legal Constraints

Because of the complexity of the $x$-ray laser system, it would be necessary to test the entire system before deploying it. The testing and deployment of the $x$-ray laser system would be a violation of:

(1) The Limited Test Ban Treaty (1963). The parties undertake "not to carry out any nuclear weapon test explosion, or any other nuclear explosion" in the atmosphere, under water, or in outer space, or in any other environment.

(2) The Treaty to Limit Anti-ballistic Missile Systems (1972). Both parties agreed to limit qualitative improvement of their ABM technology, e.g. not to develop, test or deploy ABM launchers capable of launching more than one interceptor missile at a time. "Each Party undertakes not to deploy ABM systems or their components except that..." (one site with 100 ABM interceptor missiles, 100 ABM launchers, and specified ABM radars).

(3) Outer space Treaty (1967). This forbids the placing of nuclear weapons (or other weapons of mass destruction) in orbit around the Earth and it prohibits the use of outer space for conventional military purposes.

(4) Complete Test Ban (CTB) and Anti-Satellite (ASAT) Treaties.

IX. Conclusions

In this paper we have discussed a number of scientific, technical, deployment, and legal issues that affect the viability of the $x-r a y$ laser pumped with a nuclear explosion. We have indicated in Sec. II that there are a number of fundamental difficulties that must be overcome in order for the $x$-ray laser to be a weapon with (l) high efficiency and (2) good resolution. In spite of these difficulties, we have assumed a total system efficiency of $0.1 \%$ and theoretical angular resolution (Sec. III) for our calculations of energy deposited on a target. Our results in Sec. V show that it would be necessary to have large yields (more than 0.5 megatons) to destroy a hardened missile booster, and considerably more than that for a hardened re-entry vehicle. To further complicate matters, we have indicated a number of countermeasures in. Sec. VII which could be used to overcome conceivable technical breakthroughs in the $x$-ray laser technology. When these facts are compounded together, we conclude that this system is not a feasible system to defend land based missiles or cities. 


\section{References}

1. L. Wood and G. Chapline, Nature 252, 447 (1974). G. Chapline and L. Wood, Conditions for $\dot{X}$-ray Laser Application, UCRL-75184, Lawrence Livermore Laboratory, June, 1974 . G. Chapline and L. Wood; Phys. Today 28, 40 (June, 1975). G. Chapline, Phys. Rev. A 21, $1263(1980)$.

2. M. Duguay, in Laser Induced Fusion and X-ray Laser Studies, Physics of Quantum Electronics $3 \frac{3}{(1976)}$, S. Jacobs, M. SculTy, M. Sargent, and C. Cantrell, Eds., AddisonWesley, Reading, MA.

3. R. Elton, R. Dixon, and J. Seely, in Adaptive Optics and Short Wavelength Sources, Physics of Quantum Electronics 6, 243 (i978), S. Jacobs, M. Sargent, and M. ScuIly, Eds., Addison-Wesley, Reading, MA.

4 . F. Bunkin, V. Derzhiev, and S. Yakovlenko, Sov. J. Quantum Electronics 11 , 981 (1981).

5. X-ray Laser Applications Study, S. Jorna, Ed., Defense Advanced Research Project Agency, DARPA Order 2694, JuIy, 1977 .

6. J. Smith, Science 222, 901 (1983).

7. I. Goodwin. Phys. Today 36, 43 (1983).

8. C. Robinson, Av. Week and Space Tech. 114, 25 (Feb. 23, 1981);

119,16 (oct. 17, ig83); 119, 50 (0ct. 24, ig83); 119,74 (0ct. 31, 1983); 119, 50 (Dec. 5, 1983).

9. Ballistic Missile Defense, A. Carter and D. Schwartz, Eds., Brookings, Washington, D.C., 1984 .

10. M. Gross and S. Haroche, Phys. Rept. 93, 30l (1982). J. Macgillivray and M. Feld, Contemp. Phys. 22, 299 (1981).

11. W. Bambynek, et al, Rev. Mod. Phys. 44, 716 (1972).

12. D. Matthews, et al, J. Appl. Phys. $5 \overline{4}, 4260$ (1983).

13. E. Walbridge, private communication. 1979.

14. C. Rhodes and P. Hoff, in Excimer Lasers, C. Rhodes, Ed., Springer-Verlag, New York, 